\title{
Pituitary gland metastases from renal cell carcinoma: A case report and literature update
}

\author{
Domenico Murrone ${ }^{1 *}$, Francesco Amedeo Abbate ${ }^{1}$, Danilo De Paulis ${ }^{1}$ and Renato Juan Galzio ${ }^{2}$ \\ ${ }^{1}$ Department of Neurosurgery, "San Salvatore" City Hospital, Italy \\ ${ }^{2}$ Department of Life, Health and Environmental Sciences, University of L'Aquila, Italy
}

\begin{abstract}
Pituitary metastases are rare and metastatic pituitary lesions secondary of renal carcinoma are extremely rare. These lesions can be mistaken for pituitary adenomas and diagnosis can be very difficult. We report a 67-year-old Italian female complained of headache and visual impairment resulting from a sellar lesion with suprasellar extension. A transsphenoidal biopsy was performed and histological examination revealed a metastasis of renal cell carcinoma. Few casesof this type of pituitary metastasis is described and every treatment is palliative for the life of such patient. We updateliterature and discuss the clinical and radiological features and treatment of this case.
\end{abstract}

Abbreviations: CT: Computed Tomography, MRI: Magnetic Resonance

\section{Introduction}

Metastatic tumors involving the pituitary gland account for $1 \%$ of all pituitary tumor resections and for $1 \%$ to $25 \%$ at autopsy. Any malignant tumour can metastasize to the pituitary gland, but breast and lung cancer are the most prevalent, accounting for two thirds of the cases [1-3]. McCormik described a surgical series of 780 cases of transsphenoidal surgery with only six cases $(0,8 \%)$ of pituitary secondary lesions [4]. Metastasis of renal cell carcinoma is extremely rare. Komninos reported a series of 360 cases in which $2.6 \%$ of metastatic pituitary lesions were secondary spread of renal carcinoma [5]. We present a case of a renal carcinoma metastatic to the pituitary gland and an updated review of the literature, discussing clinical and radiological characteristics of these lesions.

\section{Case report}

We present a 67-year-old Italian female complained of severe headache and visual impairment. For increasing of headache she was transferred to our department. There was no history of trauma or other systemic disease. Ophtalmological examination revealed deteriorating right vision and bitemporal hemianopsy. Ocular motion was normal and there was neither paresis nor sensory disturbance of the extremities. Endocrinological study indicated panhypopituitarism. Magnetic resonance (MR) imaging showed on T1-weighted image an isointense intrasellar mass with suprasellar extension (Figure 1), which compression of the optic chiasm and heterogeneous enhancement after administration of gadolinium. MR angiogram detected displacement of the carotid siphon bilaterally with normal signal intensity of flow. Computed tomography (CT) scan demonstrated clear demineralization and ballooning of the sellar floor and upper clivus (Figure 2). The patient underwent a trans-sphenoidal tumor biopsy (Figure 3A-3C). Histological examination revealed a metastasis of renal cell carcinoma. Post-operatively CT scan abdomen revealed renal mass without signs of other metastatic site. She underwent a right radical nephrectomy, radiotherapy with local irradiation of 41 Grey to the pituitary region and replacement hormonal therapy (Table 1).

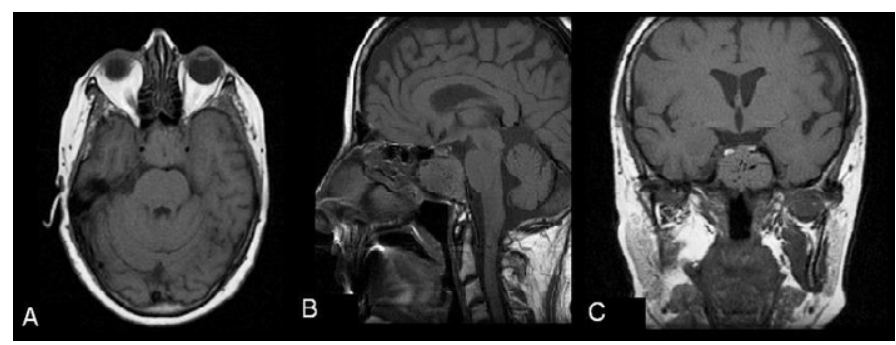

Figure 1: Axial (A), sagittal (B) and coronal (C) T1 weighted magnetic resonance (MR) images showing an isointense intrasellar mass with suprasellar extension.

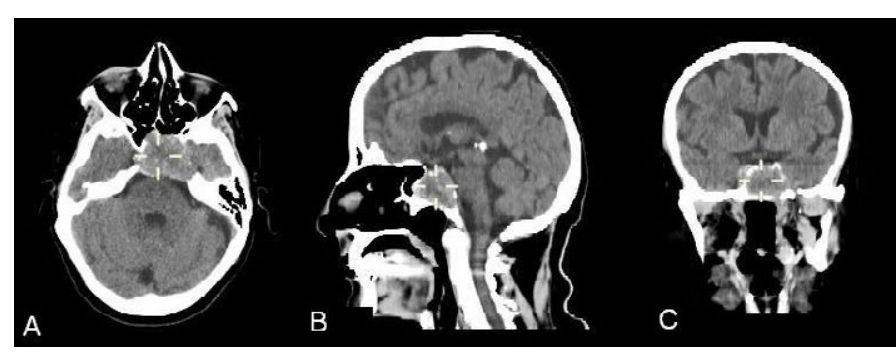

Figure 2: Axial (A), sagittal (B) and coronal (C) computed tomography (CT) scans demonstrating bony destruction of thehypophyseal fossa and upper clivus.

Correspondence to: Domenico Murrone, Department of Neurosurgery, "San Salvatore" City Hospital, via Vetoio, Coppito, L'Aquila 67100, Italy, E-mail: doflamingo82@gmail.com

Key words: pituitary metastases, renal cell carcinoma, pituitary gland, transsphenoidal biopsy

Received: August 02, 2015; Accepted: August 25, 2015; Published: August 27, 2015 
Table 1. Reported cases of pituitary metastases from renal cell carcinoma.

\begin{tabular}{|c|c|c|c|c|c|}
\hline Author (Year) & Age/Sex & Symptom & Endocrinological finding & Radiological finding & Other metastases \\
\hline Anniko et al. [6] & $59 / \mathrm{M}$ & $\begin{array}{c}\text { Weakness, } \\
\text { impotence, } \\
\text { visual impairment }\end{array}$ & Hypopituitarism & Bony destruction & Controlateral kidney \\
\hline Buonaguidi et al. [1] & $53 / \mathrm{M}$ & $\begin{array}{c}\text { Weakness, } \\
\text { visual impairment }\end{array}$ & $\begin{array}{l}\text { Hypopituitarism, } \\
\text { diabete insipidus }\end{array}$ & $\begin{array}{l}\text { Bony destruction, } \\
\text { enhanced }\end{array}$ & NR \\
\hline James et al. [2] & $75 / \mathrm{M}$ & Visual impairment & Normal & $\begin{array}{l}\text { Bony destruction, } \\
\text { enhanced }\end{array}$ & NR \\
\hline Eick et al. [10] & $66 / \mathrm{M}$ & Weakness & Hypopituitarism & enhanced & NR \\
\hline Horikoshi et al. [12] & $51 / \mathrm{M}$ & $\begin{array}{c}\text { Headache, } \\
\text { visual impairment }\end{array}$ & Hypopituitarism & $\begin{array}{l}\text { Bony destruction, } \\
\text { enhanced }\end{array}$ & Lung \\
\hline McCormick et al. [4] & $35 / \mathrm{F}$ & Visual impairment & Hypopituitarism & $\begin{array}{c}\text { Bony destruction, } \\
\text { enhanced }\end{array}$ & NR \\
\hline Koshiyama et al. [13] & $57 / \mathrm{M}$ & Visual impairment & $\begin{array}{l}\text { Hypopituitarism, } \\
\text { diabete insipidus }\end{array}$ & enhanced & Pancreas \\
\hline Nishio [16] & $63 / \mathrm{F}$ & Visual impairment & Hypopituitarism & enhanced & NR \\
\hline Weiss et al. [21] & $59 / \mathrm{M}$ & $\begin{array}{l}\text { Lethargy, impotence, visual } \\
\text { impairment }\end{array}$ & Hypopituitarism & $\begin{array}{l}\text { Bony destruction, } \\
\text { enhanced }\end{array}$ & NR \\
\hline Uchino et al. [18] & $63 / \mathrm{F}$ & Visual impairment & NR & enhanced & NR \\
\hline Beckett et al. [8] & $56 / \mathrm{M}$ & $\begin{array}{l}\text { Headache, } \\
\text { Horner's syndrome }\end{array}$ & Hypopituitarism & enhanced & NR \\
\hline \multirow[t]{2}{*}{ Marar et al. [15] } & $54 / \mathrm{M}$ & Visual impairment & Hypopituitarism & NR & NR \\
\hline & $73 / \mathrm{M}$ & Asymptomatic & hypopituitarism & NR & NR \\
\hline Weil [20] & $53 / \mathrm{M}$ & $\begin{array}{c}\text { Headache, } \\
\text { impotence, } \\
\text { 3th nerve palsy, } \\
\text { visual impairment }\end{array}$ & $\begin{array}{l}\text { Hypopituitarism, } \\
\text { diabete insipidus }\end{array}$ & enhanced & NR \\
\hline Weber et al. [19] & $62 / \mathrm{F}$ & Headache, Visual impairment & Normal & enhanced & NR \\
\hline Djimi et al. [9] & $45 / \mathrm{M}$ & Visual impairment & Normal & enhanced & Lung \\
\hline Basaria et al. [7] & $77 / \mathrm{F}$ & Visual impairment & Hypopituitarism & NR & NR \\
\hline Yokoyama et al. [3] & $63 / \mathrm{M}$ & $\begin{array}{c}\text { Headache, } \\
\text { Visual impairment }\end{array}$ & $\begin{array}{l}\text { Hypopituitarism, } \\
\text { diabete insipidus }\end{array}$ & $\begin{array}{l}\text { Bony destruction, } \\
\text { enhanced }\end{array}$ & Lung \\
\hline Pallud et al. [17] & $70 / \mathrm{M}$ & Visual impairment & NR & NR & NR \\
\hline Liu et al. [14] & $54 / \mathrm{M}$ & Visual impairment & $\begin{array}{l}\text { Hypopituitarism, } \\
\text { diabete insipidus }\end{array}$ & $\begin{array}{l}\text { Bony destruction, } \\
\text { enhanced }\end{array}$ & NR \\
\hline \multirow[t]{5}{*}{ Gopan et al. [11] } & $67 / \mathrm{M}$ & Visual impairment & $\begin{array}{l}\text { Hypopituitarism, } \\
\text { diabete insipidus }\end{array}$ & NR & NR \\
\hline & $51 / \mathrm{M}$ & Visual impairment & hypopituitarism & NR & NR \\
\hline & $53 / \mathrm{M}$ & Visual impairment & $\begin{array}{l}\text { Hypopituitarism, } \\
\text { diabete insipidus }\end{array}$ & NR & NR \\
\hline & $67 / \mathrm{F}$ & Asymptomatic & Hypopituitarism & NR & NR \\
\hline & $61 / \mathrm{F}$ & Asymptomatic & Hypopituitarism & NR & NR \\
\hline Present case & $67 / \mathrm{F}$ & $\begin{array}{c}\text { Headache, } \\
\text { Visual impairment }\end{array}$ & Hypopituitarism & $\begin{array}{l}\text { Bony destruction, } \\
\text { enhanced }\end{array}$ & Absent \\
\hline
\end{tabular}
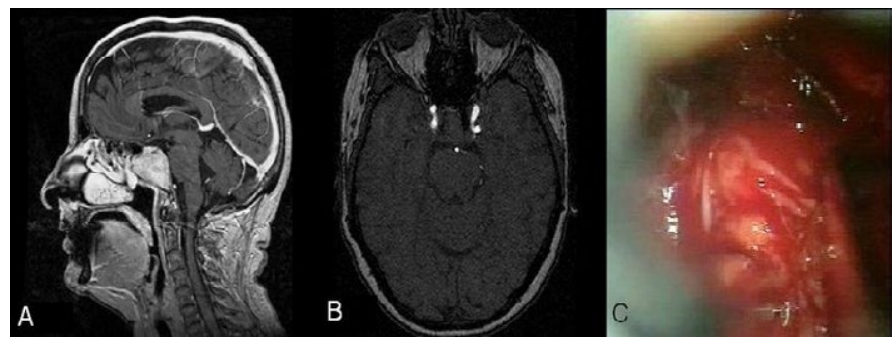

Figure 3: A) Sagittal T1 weighted MR image with gadolinium revealing marked contrast enhancement of the tumor without identifiable normal pituitary gland. B)Post-contrast axial T1 weighted MR angiogram showing displacement of the carotid siphon bilaterally with normal signal intensity of flow B) Intra-operative photograph of intrasellare mass.

\section{Discussion}

Symptomatic pituitary metastasis of renal cell carcinoma is very rare and only 25 cases have been described [1-4,6-21]. Only $7 \%$ of pituitary metastases are symptomatic $[13,19,22]$. Clinically diabete insipidus and visual field impairment are more frequent in pituitary metastasis than in pituitary adenoma, for involving of the posterior lobe of gland and the optic chiasm $[6,16,20]$. Gopan suggested pituitary metastases from renal cell carcinoma behave differently from other metastatic malignancies, more commonly involving the anterior pituitary [11]. In these cases hypopituitarism is more frequent than diabete insipidus. Cranial neuropathy can be present $[8,12,15,20]$. Radiological findings of pituitary metastases are very similar to those of primary pituitary tumor. On CT scan there are not important differences. MRI is not specific for pituitary metastases but particular signs, as isointensity on both $\mathrm{T} 1$ and $\mathrm{T} 2$ weighted images and loss of high intensity signal from posterior lobe of gland, can be suggestive $[4,6,7,9,10,16-18,21]$.

Liu et al discovered that in many cases of pituitary metastasis there are a strong enhancement of the mass and a bone destruction without marked sellar enlargement [14]. McCutcheon et al. discovered that increased signal intensity in the contiguous hypothalamus on 
T2-weighted MR images is frequently observed in these metastases [23]. Thickening of the pituitary stalk, sclerosis of the surrounding sellaturcica and invasion of the cavernous sinus may indicate a secondary lesion [24]. In some cases the diagnosis of the primary neoplasm was performed after the diagnosis of pituitary lesion. Rarely is it possible a total or subtotal surgical removal of the mass, while it is always recommended diagnostic biopsy. Chemotherapeutic agents like sorafenib and sunitinib can be useful [11]. The long-term benefit of postoperative radiotherapy is not known due to the rarity of such cases while stereotactic radiotherapy can be beneficial in sparing the optic nerves [22].

\section{Conclusion}

Pituitary metastases from renal cell carcinoma are very rare and may be difficult to diagnose. Clinical and radiological features may be suggestive. These lesions usually occur in highly disseminated renal cell carcinoma and every treatment can contribute to the improvement of the quality of life of such patients.

\section{Consent}

Written informed consent was obtained from the patient for publication of this case report and any accompanying images.

\section{References}

1. Buonaguidi R, Ferdeghini M, Faggionato F, Tusini G (1983) Intrasellar metastasis mimicking a pituitary adenoma. Surg Neurol 20: 373-378. [Crossref]

2. James RL Jr, Arsenis G, Stoler M, Nelson C, Baran D (1984) Hypophyseal metastatic renal cell carcinoma and pituitary adenoma. Case report and review of the literature. Am J Med 76: 337-340. [Crossref]

3. Yokoyama T, Yoshino A, Katayama Y, Watanabe T, Kashima Y, et al. (2004) Metastatic pituitary tumor from renal cell carcinoma treated by fractionated stereotactic radiotherapy--case report. Neurol Med Chir (Tokyo) 44: 47-52. [Crossref]

4. McCormick PC, Post KD, Kandji AD, Hays AP (1989) Metastatic carcinoma to the pituitary gland. Br J Neurosurg 3: 71-79. [Crossref]

5. Komninos J, Vlassopoulou V, Protopapa D, Korfias S, Kontogeorgos G, et al. (2004) Tumors metastatic to the pituitary gland: case report and literature review. $J$ Clin Endocrinol Metab 89: 574-580. [Crossref]

6. Anniko M, Lundquist PG, Silfverswärd C, Wersäll J (1981) Hypernephroma metastasis in the pituitary gland. A case report. Arch Otorhinolaryngol 232: 227-232. [Crossref]

7. Basaria S, Westra WH, Brem H, Salvatori R (2004) Metastatic renal cell carcinoma to the pituitary presenting with hyperprolactinemia. J Endocrinol Invest 27: 471-474. [Crossref]
8. Beckett DJ, Gama R, Wright J, Ferns GA (1998) Renal carcinoma presenting with adrenocortical insufficiency due to a pituitary metastasis. Ann Clin Biochem 35 : 542544. [Crossref]

9. Djimi H, Donnio A, Ayeboua L, Vally P, Olindo S, et al. (2003) Hypophysis metastasis of a hypernephroma tumor revealed by a chiasma syndrome. J Fr Ophtalmol 26: 976979. [Crossref]

10. Eick JJ, Bell KA, Stephan MT, Fuselier HA Jr (1985) Metastatic renal cell carcinoma presenting as an intrasellar mass on computerized tomography. J Urol 134: 128-130. [Crossref]

11. Gopan T, Toms SA, Prayson RA, Suh JH, Hamrahian AH, et al. (2007) Symptomatic pituitary metastases from renal cell carcinoma. Pituitary 10: 251-259. [Crossref]

12. Horikoshi T, Mitsuka S, Kimura R, Fukamachi A, Nukui H (1988) Renal cell carcinoma metastatic to the hypophysis--case report. Neurol Med Chir (Tokyo) 28: 7882. [Crossref]

13. Koshiyama H, Ohgaki K, Hida S, Takasu K, Yumitori K, et al. (1992) Metastatic renal cell carcinoma to the pituitary gland presenting with hypopituitarism. $J$ Endocrinol Invest 15: 677-681. [Crossref]

14. Liu H, Yamaki T, Oka S, Koyanagi I, Houkin K (2005) Metastatic renal cell carcinoma mimicking pituitary adenoma: case report. Neurol Med Chir (Tokyo) 45: 418-422. [Crossref]

15. Marar IE, Kandil H, Kanal E, Marion D, Inman M, et al. (1998) Renal cell carcinoma metastatic to the pituitary gland: clinical manifestations and successful treatment with transsphenoidal resection. Endocr Pract 4: 204-207. [Crossref]

16. Nishio S, Tsukamoto H, Fukui M, Matsubara T (1992) Hypophyseal metastatic hypernephroma mimicking a pituitary adenoma. Case report. Neurosurg Rev 15: 319322. [Crossref]

17. Pallud J, Nataf F, Roujeau T, Roux FX (2005) Intraventricular haemorrhage from a renal cell carcinoma pituitary metastasis. Acta Neurochir (Wien) 147: 1003-1004 [Crossref]

18. Uchino A, Hasuo K, Mizushima A, Matsumoto S, Mihara F, et al. (1996) Intracranial metastasis of renal cell carcinoma: MR imaging. Radiat Med 14: 71-76. [Crossref]

19. Weber J, Gassel AM, Hoch A, Spring A (2003) Concomitant renal cell carcinoma with pituitary adenoma. Acta Neurochir (Wien) 145: 227-231. [Crossref]

20. Weil RJ (2002) Pituitary metastasis. Arch Neurol 59: 1962-1963. [Crossref]

21. Weiss RE, Corvalan AH, Dillon RW (1993) Metastatic renal cell carcinoma presenting as impotence. J Urol 149: 821-822. [Crossref]

22. Morita A, Meyer FB, Laws ER Jr (1998) Symptomatic pituitary metastases. J Neurosurg 89: 69-73. [Crossref]

23. McCutcheon IE, Kitagawa RH, Sherman SI, Bruner JM (2001) Adenocarcinoma of the salivary gland metastatic to the pituitary gland: case report. Neurosurgery 48: 11611165. [Crossref]

24. Fassett DR, Couldwell WT (2004) Metastases to the pituitary gland. Neurosurg Focus 16: E8. [Crossref]

Copyright: (C) 2015 Morris TM. This is an open-access article distributed under the terms of the Creative Commons Attribution License, which permits unrestricted use, distribution, and reproduction in any medium, provided the original author and source are credited. 